\title{
La conscience auto-organisatrice : une alternative au modèle dominant de la psychologie cognitive
}

Pierre Perruchet, Annie Vinter et Sébastien Pacton

\section{(2) OpenEdition}

\section{Édition électronique}

URL : http://journals.openedition.org/educationdidactique/224

DOI : 10.4000/educationdidactique.224

ISBN : 978-2-7535-1616-8

ISSN : $2111-4838$

\section{Éditeur}

Presses universitaires de Rennes

\section{Édition imprimée}

Date de publication : 1 décembre 2007

Pagination : 105-116

ISBN : 978-2-7535-0597-1

ISSN : 1956-3485

\section{Référence électronique}

Pierre Perruchet, Annie Vinter et Sébastien Pacton, « La conscience auto-organisatrice : une alternative au modèle dominant de la psychologie cognitive », Éducation et didactique [En ligne], 1-3 | Décembre 2007, mis en ligne le 01 décembre 2009, consulté le 09 décembre 2020. URL : http:// journals.openedition.org/educationdidactique/224 ; DOI : https://doi.org/10.4000/ educationdidactique.224 


\title{
LA CONSCIENCE AUTO-ORGANISATRICE : UNE ALTERNATIVE AU MODÈLE DOMINANT DE LA PSYCHOLOGIE COGNITIVE
}

\author{
Pierre Perruchet, Annie Vinter, Université de Bourgogne, LEAD \\ Sébastien Pacton, Université Paris Descartes, LPNC
}

\begin{abstract}
Résumé : Il est généralement supposé que les opérations cognitives auxquelles nous n'avons aucun accès introspectif reposent sur des représentations et des formes de raisonnement identiques à celles qui composent la pensée consciente. Cet article présente une vision alternative, dans laquelle il n'y a plus de place pour un «inconscient cognitif». Ladaptation repose sur la formation de représentations conscientes, dont la remarquable organisation émerge naturellement grâce à des processus élémentaires d'auto-organisation, par le jeu des interactions dynamiques entre le sujet et son environnement. Quelques pistes de réflexion sont suggérées quant aux enjeux de cette perspective pour l'éducation.
\end{abstract}

Mots clés : conscience, auto-organisation, règle, régularités statistiques, développement

Perruchet \& al

Il est évident pour chacun que, confronté par exemple au choix d'une nouvelle voiture, nous partons de connaissances explicites, sur l'état du marché, les caractéristiques des véhicules, les possibilités de contracter un emprunt, etc., et nous raisonnons en pesant l'ensemble des arguments pour aboutir à ce qui nous apparaîtra comme étant la meilleure solution. Il en est de même pour des comportements plus anodins. Ainsi lorsqu'il s'agit de déterminer l'orthographe de mots phonologiquement identiques (e.g., rayer, rayé, ou rayés?), nous nous appuyons, au moins en certaines occasions, sur des règles explicites (e.g., s'il s'agit d'un adjectif et si le nom est masculin pluriel, l'orthographe correcte sera « rayés" pour respecter les règles d'accord). Il est non moins évident toutefois que les raisons sous-tendant le comportement ne sont pas toujours accessibles à un examen introspectif. Pour rester sur l'exemple de l'orthographe, les règles d'accord sont souvent respectées sans que ceci ne semble lié à une délibération explicite de la part du scripteur. Plus clairement encore, il est bien difficile de rapporter quels enchaînements de processus conduisent à capturer le sens d'un mot ou d'une phrase.

Une question fondamentale est de savoir si le même genre de processus est impliqué selon que ceux-ci donnent prise, ou non, à un accès introspectif. La réponse dominante proposée par la psychologie cognitive depuis bientôt un demisiècle est clairement positive. La cognition humaine procéderait à partir de propositions symboliques sur lesquelles s'appliquent les ressources du raisonnement et de la logique, que l'on ait ou non conscience de procéder ainsi. Dans ce contexte, si le jeune enfant dit correctement " une belle chanson * plutôt que « un beau chanson », c'est parce qu'il sait que « beau » est un adjectif, « chanson » un nom, et que l'adjectif doit s'accorder en genre avec le nom. Le fait qu'il soit encore incapable de formuler les règles de la grammaire ne change rien. Ainsi l'idée selon laquelle la majorité des opérations cognitives repose sur la connaissance de règles inconscientes, et sur l'exploitation de ces connaissances au moyen de raisonnements et inférences également inconscients, est largement admise par la communauté scientifique.

Cette conception peut apparaître assez naturelle. Cela fait longtemps que l'idée d'un « inconscient affectif " fonctionnant sur le mode de la pensée consciente, mais sans accès introspectif immédiat, s'est répandue dans les esprits par le biais de la psychanalyse. L' « inconscient cognitif » postulé par la psychologie cognitive relève de la même démarche générale. Mais on peut également s'interroger sur la cohérence générale d'un modèle fonctionnel ainsi structuré. Par exemple, si les mêmes processus peuvent se dérouler à un niveau conscient et à un niveau inconscient, pourquoi l'évolution a-t-elle conduit à l'émergence de la conscience? À quoi la conscience sert-elle exactement? N'existerait-il pas 
des modèles plus parcimonieux, évitant cette sorte de « dédoublement » de l'esprit?

\section{Le principe général d'une alternative}

Lobjectif de cet article est de présenter une position alternative, celle défendue par les auteurs dans différents supports (e.g., Perruchet \& Vinter, 2002, sous presse). Largument est, en résumé, le suivant. Il ne s'agit pas de nier que nous recourrns à des raisonnements et inférences sur la base de connaissances conscientes à chaque fois que cette façon de procéder correspond à notre expérience vécue. Là s'arrêterait, toutefois, la pertinence de cette explication. Le mode de traitement dont nous avons l'expérience phénoménale immédiate, à notre sens, ne peut servir de modèle à des mécanismes inconscients. Lorsque l'expérience phénoménale de réaliser des opérations mentales fait défaut, les processus mis en jeu seraient d'un ordre totalement différent. En bref, en lieu et place d'opérations logiques inconscientes portant sur des unités élémentaires plus ou moins invariables, nous développerions par apprentissage des unités représentationnelles conscientes, dont la complexité irait croissante.

Dans quelle mesure la formation d'unités conscientes peut-elle se substituer à des opérations symboliques inconscientes? Pour commencer par un exemple simpliste, il paraît légitime de penser qu'énoncer « une belle chanson » ne nécessite pas nécessairement une analyse des catégories syntaxiques et une connaissance des règles d'accord. C'est peut-être plus simplement parce qu'au lieu de développer d'une part un lexique dans lequel les mots sont stockés de façon indépendante, et d'autre part une grammaire permettant de régir les relations entre ces mots (c'est-à-dire de procéder à la même analyse que le grammairien), nous formons directement des unités de plus haut niveau. Peut-être l'enfant parlet-il d' " une belle chanson» parce qu'il a mémorisé cette expression telle quelle, et qu'il n'a par ailleurs jamais entendu dire « un beau chanson».

Prenons un autre exemple, dans le domaine de la résolution de problèmes, portant plus précisément sur la notion de transitivité. Dans les tâches d'ordonnancement linéaire, deux prémisses sont présentées, dont l'expression formelle peut être : A est plus long que $\mathrm{B}$, et $\mathrm{B}$ est plus long que $\mathrm{C}$. On demande aux sujets de juger si l'expression «A est plus long que $C$ » est correcte. On peut bien sûr supposer que les sujets qui résolvent cette tâche possèdent une connaissance formelle de la notion de transitivité, et qu'ils savent que cette notion s'applique à la relation "plus long que ». À nouveau, si c'est effectivement ce que les sujets ont conscience de faire, rien ne permet de rejeter l'hypothèse. Si par contre il s'agit, par exemple, d'enfants n'ayant jamais entendu parler de transitivité, pourquoi considérer qu'ils opèrent inconsciemment le même raisonnement? Une solution plus simple consiste à supposer qu'ils ont construit une représentation intégrée des prémisses sous un format analogique, et qu'ils lisent la réponse directement sur cette représentation. Si l'on se forme l'image des trois bâtons alignés, A, B, et $\mathrm{C}$, nul besoin de raisonner pour dire que $\mathrm{A}$ est plus long que $C$. Notons que pour ce genre de tâche, il y a consensus autour de l'idée selon laquelle l'on procède effectivement de cette façon (Evans et al. 1993).

Ces exemples illustrent une idée fondamentale, à savoir qu'une représentation isomorphe à la structure du monde peut se substituer à la connaissance de règles. Cette idée, qui est fondamentale pour notre cadre théorique, ne nous est pas propre. Elle a été longuement développée, en particulier, par Clark et Thornton (1997). Ces auteurs montrent comment un changement relativement mineur (bien que toujours dans le sens d'une complexité accrue) dans le mode de codage d'un problème peut conduire à simplifier de façon qualitative la résolution de ce problème. Ils évoquent à ce propos la notion de « representation/ computation trade-off $»$ : la complexité computationnelle décroît dans des proportions considérables lorsque les représentations de base deviennent plus élaborées.

\section{Le problème à résoudre}

Mais y a t-il vraiment là les germes d'une remise en cause du modèle prédominant de la psychologie cognitive, fondé sur l'existence d'opérations symboliques inconscientes? Il faut reconnaître que les exemples ci-dessus ne concernent que quelques cas simples. Pour ne reprendre que le premier, il est certes facile d'admettre que l'énoncé « une belle chanson » peut être basé sur la mémorisation d'une 
représentation et non sur la connaissance de règles d'accord adjectif/verbe, mais qu'en est-il de constructions syntaxiques beaucoup plus élaborées? Peut-être peut-on envisager une explication en imaginant la formation d'unités cognitives très complexes. Mais alors un second problème apparaît : comment expliquer la formation de ces unités cognitives ellesmêmes? Si la formation de ces unités requiert des processus d'abstraction inconsciente aussi complexes que ceux postulés dans l'approche traditionnelle, le gain paraît bien mince.

Ainsi la gageure consiste-t-elle à expliquer comment la construction d'unités cognitives isomorphes au monde réel, suffisamment élémentaires pour qu'il soit possible d'en expliquer la formation par des mécanismes biologiquement plausibles, peut rendre compte de comportements adaptés extraordinairement complexes, tels que, par exemple, ceux qui sont mis en jeu dans la compréhension et la production du langage. C'est là le cœur de notre contribution. Nous en présenterons l'idée générale en deux temps. Il s'agira d'abord de montrer que des mécanismes extrêmement simples peuvent rendre compte de la formation d'unités cognitives relativement complexes. Dans un second temps, nous examinerons pourquoi ces unités relativement complexes (le "relativement» suggérant qu'une partie seulement du problème est résolue) pourraient en fait se révéler suffisantes pour expliquer des performances reposant en apparence sur des connaissances très élaborées.

Une remarque préalable paraît utile. Les explications proposées dans les deux sections suivantes pourront paraître assez alambiquées, et l'on peut se demander au préalable si le modèle cognitif conventionnel ne fournit pas une explication plus parcimonieuse. Ceci est sans doute vrai, mais dans un sens seulement. Les chercheurs ont naturellement une vision faussée de la parcimonie d'une explication, car ils l'évaluent à l'aune de leur propre difficulté de compréhension, plutôt que de considérer la plausibilité des mécanismes sollicités chez le sujet. Or il convient de soigneusement distinguer à ce niveau le point de vue de l'observateur et celui du sujet, car la simplicité de l'explication pour l'observateur semble varier souvent en raison inverse de la simplicité des mécanismes impliqués chez l'apprenant. C'est le cas ici. L'explication cognitiviste traditionnelle est simple pour l'observateur - chacun peut comprendre, par exemple, comment la connaissance inconsciente de règles grammaticales permet de formuler des énoncés corrects - mais elle requiert de la part du sujet des capacités d'abstraction extraordinairement complexes. Lexplication que nous proposons a les propriétés exactement inverses. Elle est sans doute plus complexe à comprendre, mais elle ne requiert de l'apprenant que des capacités élémentaires.

\section{Comment se forment les unités cognitives?}

Il s'agit donc d'abord de montrer la capacité de mécanismes élémentaires à élaborer des représentations cognitives isomorphes au monde réel. La formation d'unités mentales en elle-même peut être expliquée très simplement en recourrant aux principes élémentaires de l'apprentissage associatif. La présentation simultanée et répétée de deux événements initialement indépendants, $\mathrm{A}$ et $\mathrm{B}$, conduit naturellement à la formation d'une unité $\mathrm{AB}$. Un nombre considérable de recherches conduisent à l'idée selon laquelle la condition nécessaire et suffisante pour la formation de cette unité est le traitement attentionnel conjoint de A et de B (Pacton $\&$ Perruchet, sous presse). Ceci signifie qu'il n'y a, par exemple, nul besoin d'une quelconque «intention » d'apprendre : le lien se forme automatiquement dès lors que les événements sont traités conjointement dans un même foyer attentionnel. Pour cette raison, cette forme d'apprentissage est souvent qualifiée d' « implicite ».

S'il est aisé de comprendre comment l'apprentissage implicite peut conduire à la formation de nouvelles unités cognitives, le recours à des principes associatifs élémentaires semble toutefois limiter a priori la puissance explicative de l'interprétation. Comment se fait-il que les unités cognitives ainsi formées soient isomorphes aux unités constitutives du monde réel, permettant au sujet de se construire une représentation suffisamment fidèle du monde dans lequel il vit?

\section{La notion d'auto-organisation}

Lexplication recourt ici à la notion d'auto-organisation. Le concept d'auto-organisation a été beaucoup utilisé pour rendre compte de l'organisation 
remarquable de certains comportements dans les sociétés d'insectes. Considérons un exemple particulier, relatif au recrutement alimentaire chez les fourmis. Parmi plusieurs chemins possibles vers une source de nourriture, les fourmis tendent à choisir le plus court. Comment un tel comportement collectif adapté est-il possible? Une explication calquée sur les modèles cognitivistes standards pourrait être que les fourmis ont mesuré différents chemins, et ont délibérément choisi le plus court sur la base d'une comparaison exhaustive des mesures. Mais il est bien difficile de prêter les capacités requises aux fourmis, avec leurs quelques milliers de neurones pour un volume cérébral d'environ $1 \mathrm{~mm} 3$. Fort heureusement, les biologistes des comportements sociaux de sociétés d'insectes ont proposé un autre modèle (e.g. Deneubourg, Aron, Goss, \& Pasteels, 1990). Dans ce modèle, les fourmis partent au hasard dans toutes les directions, et ce faisant découvrent de la nourriture plus ou moins rapidement. Elles retournent alors au nid en traçant une piste chimique constituée de phéromones. Ces traces odorantes vont agir comme signaux pour inciter d'autres ouvrières à emprunter le même chemin, lesquelles y déposeront à leur tour des phéromones. Après un certain temps, les fourmis tendront à converger sur le chemin le plus direct, car les fourmis qui ont découvert par hasard le chemin le plus court retourneront plus vite au nid. Ainsi dans un temps donné, ce chemin est parcouru plus souvent que les autres, ce qui entraîne une plus grande concentration de phéromones sur le trajet et donc un pouvoir d'attraction supérieur. Dans le même temps, les autres traces de phéromones s'évaporent, renforçant la valeur relative du chemin optimal.

Ainsi les fourmis aboutissent-elles à une organisation finale optimale, sans poursuivre aucun plan prédéterminé ni posséder aucune « connaissance » relative à la longueur des chemins, ce qui justifie la description de ces comportements collectifs comme résultant de processus d'auto-organisation. C'est de l'interaction directe entre certains comportements élémentaires des fourmis (leur propension à suivre et à renforcer un chemin marqué par des phéromones) et certaines propriétés du monde physique (un chemin plus court qu'un autre est parcouru plus souvent en un laps de temps donné, les phéromones tendent à s'évaporer avec le temps) que résulte le comportement collectif auto-organisé des fourmis.

\section{La formation des mots}

Comment ce type d'explication peut-il se transposer à la formation d'unités cognitives? Il ne s'agit pas d'assimiler les fourmis aux neurones, par exemple, mais de considérer que la formation d'unités conscientes isomorphes au monde réel représente un mode d'adaptation comparable à la remarquable organisation d'une fourmilière, et que le mode général d'explication proposé pour les secondes pourrait également s'appliquer aux premières. L'idée fondamentale à reprendre est celle d'une interaction dynamique (i.e. au cours du temps) entre les propriétés de l'organisme et les propriétés de l'environnement. Pour développer le principe sur un exemple, prenons celui de l'extraction des mots du langage par le bébé.

Pour le bébé, les phrases apparaissent essentiellement comme une suite ininterrompue de sons: les transitions entre mots ne sont en effet marquées par aucune interruption dans le signal acoustique. Comment le bébé va-t-il apprendre que « voilatonbiberon » est composé de trois mots? Une partie de la réponse semble être qu'il tire parti des régularités statistiques du langage. Si l'on considère toutes les partitions possibles de la chaîne verbale (voi, voila, voilaton, laton, etc.), les mots (voilà, ton, biberon) reviendront en moyenne plus fréquemment dans l'ensemble du discours perçu par l'enfant que les unités chevauchant plusieurs mots (laton, tonbi). Une analyse statistique permet donc de retrouver (au moins en partie) les unités pertinentes (les mots) d'un flux continu de parole. Il existe bien évidemment des algorithmiques mathématiques permettant de faire les calculs nécessaires (e.g., Brent, 1996). Toutefois, il est également possible de retrouver les mots très simplement, grâce à des processus d'autoorganisation en de nombreux points similaires à ceux impliqués dans la recherche de nourriture par les fourmis. La recherche de nourriture chez les fourmis commence par une exploration aléatoire. De la même façon, on peut imaginer que le bébé commence par percevoir des unités provisoires, composées de façon aléatoire d'une ou quelques syllabes. Cette segmentation du matériel auditif en unités de tailles réduites peut être pensée comme une conséquence obligatoire des limites du traitement attentionnel. Bien évidemment, ces unités peuvent correspondre à des mots, mais dans le cas général, elles ont plus de chance d'être erronées, comme les chemins initialement 
empruntés par les fourmis ont plus de chance d'être inadaptés. La sélection du bon chemin par les fourmis va s'opérer grâce au fait qu'en un temps donné, les allers-retours sur le chemin le plus court seront plus nombreux. La sélection des mots peut s'opérer sur un mode similaire. Si le bébé a formé les unités « voila » ou « biberon », il a de fortes chances de les réentendre fréquemment par la suite : elles apparaîtront dans des phrases différentes, comme « voilà le chien » ou «le biberon est chaud». Les conditions sont donc remplies pour que ces unités se renforcent en mémoire grâce à leur répétition. Si par contre c'est « laton» qui a été initialement perçu, cette unité a de bonnes chances de disparaître par oubli et interférence, car «laton» sera perçu moins souvent que chacun des mots dont il emprunte une syllabe.

Ainsi donc, les mots peuvent émerger d'un flux continu de discours grâce aux lois élémentaires de la mémoire et de l'apprentissage associatif. La formation initiale d' " unités candidates » se fait naturellement, par la perception dans un même foyer attentionnel de quelques syllabes adjacentes. L'oubli et l'interférence conduisent ensuite à supprimer les moins fréquentes de ces unités candidates, et les propriétés du langage font que les unités résiduelles ont de fortes chances d'être des mots, parce qu'elles sont les plus fréquentes du discours. C'est de l'interaction directe entre des propriétés du fonctionnement attentionnel et mnésique du bébé (un empan attentionnel limité, une propension à oublier ce qui est peu souvent répété) et des propriétés du matériau linguistique (les successions de syllabes composant les mots se répètent davantage que les successions de syllabes chevauchant les frontières de mots) dont résulte la capacité à identifier les mots de la langue.

Ce schéma explicatif a été testé au moyen d'une modélisation informatique portant sur un microlangage artificiel (Perruchet \& Vinter, 1998a). Les simulations effectuées suggèrent qu'il s'agit là d'une procédure extrêmement efficace. On voit donc sur cet exemple comment des procédures d'auto-organisation ne requérant que des capacités élémentaires de la part de l'apprenant peuvent le conduire à former rapidement des unités représentatives du monde perçu. Si des outils mathématiques sophistiqués parviennent au même résultat, il faut noter qu'ils ne sont en aucun cas nécessaires.

\section{Vers un modèle général : la Conscience Auto-Organisatrice}

L'exemple développé jusqu'à présent illustre comment on peut expliquer la formation d'unités conscientes isomorphes au monde réel de façon très économique, grâce aux principes d'auto-organisation. De façon plus formelle, l'explication que nous proposons repose sur l'idée selon laquelle l'isomorphisme entre les représentations mentales conscientes du sujet et la structure de son environnement est le produit final d'une organisation progressive qui émerge, au cours du temps, grâce à l'action de processus élémentaires associatifs prenant les représentations conscientes elles-mêmes comme objets sur lesquels opérer. Ce faisant, la conscience participe activement à son propre développement, d'où la notion de conscience auto-organisatrice.

En résumé, le contenu de l'expérience consciente momentanée, nécessairement polymorphe, sollicite automatiquement le déclenchement de mécanismes associatifs élémentaires. Ceux-ci créent ou renforcent des liens entre certaines composantes, conduisant à la formation de nouvelles unités. Ces nouvelles unités guident le traitement attentionnel des sujets, et contribuent à modifier l'expérience consciente du sujet lorsque celui-ci se retrouve à nouveau confronté à une situation similaire. Ces unités nouvellement créées peuvent à leur tour entrer comme composantes dans des unités plus larges ou plus complexes, et ainsi de suite de façon itérative au fil des expériences conscientes successives. Toute la puissance de traitement du système neuronal humain serait dévolue à forger l'expérience consciente momentanée, qui assure tout à la fois l'adaptation immédiate du sujet à son environnement, et la mise en place des changements anticipant sa propre évolution vers une meilleure efficience représentationnelle.

La question est maintenant de savoir jusqu'où il est possible d'aller dans le « representation/computation trade-off » de Clark et Thornton (1997) ? Est-il possible d'arriver à supprimer toute la composante computationnelle inconsciente au profit de la formation d'unités cognitives conscientes?

\section{Pourquoi une telle puissance explicative?}

La puissance explicative de la conception proposée naît de deux considérations. La première est que le 
processus d'auto-organisation décrit plus haut permet la formation d'unités cognitives plus complexes que ce que l'on pourrait imaginer de simples mécanismes associatifs. En effet, l'attention nécessaire à la formation de ces unités peut être capturée par des événements autres que les traits de surface élémentaires de la situation. Déjà chez le bébé, des relations abstraites, certes élémentaires, comme les relations de répétition ou de symétrie, sont immédiatement perçues. Avec le développement progressif de nouvelles primitives perceptives, la complexité des unités immédiatement perçues comme telles va naturellement en s'accroissant, sans qu'il soit nécessaire de faire appel à d'autres mécanismes que les processus d'auto-organisation évoqués plus haut. Pour illustrer cet aspect par un exemple bien connu, le joueur d'échec chevronné va percevoir directement des configurations dans ce qui apparaît au débutant comme une simple juxtaposition de pièces sur l'échiquier.

Mais, comme annoncé plus haut, il y a un second volet dans l'approche. En bref, c'est le fait que ces unités perceptivement saillantes se révèlent encore plus pertinentes pour l'adaptation - y compris à des situations en apparence très complexes - qu'il n'y parait au départ. La raison en est que, bien souvent, les caractéristiques du matériel à apprendre qui deviennent perceptivement saillantes pour l'apprenant au fil de l'apprentissage sont suffisamment corrélées avec les propriétés structurales de ce matériel pour que la sensibilité à ces caractéristiques conduise à des ajustements comportementaux identiques à ceux qui seraient mis en place si la structure profonde avait été elle-même apprise. Lidée générale est donc que l'adaptation s'opère par la découverte d'aspects corrélés, parfois secondaires en apparence, qui peuvent donner prise à des processus associatifs élémentaires, et qui permettent une bonne approximation du comportement qui résulterait de la connaissance de la structure formelle du domaine.

Prenons un exemple dans le domaine de l'orthographe. Les règles d'accord en nombre, par exemple du nom et du verbe, nécessitent pour leur application une analyse syntaxique de la phrase : il faut au minimum identifier la catégorie syntaxique de chaque élément, et relier le verbe à son sujet. Or un peu d'introspection suggère que l'on peut marquer correctement l'accord sans toujours faire cette analyse. Serait-ce alors qu'il s'agit d'application inconsciente de règles, en contradiction avec les postulats posés plus haut? Pas nécessairement, car il se trouve que dans bien des cas, l'accord correct peut être formé en prenant en compte des régularités superficielles relativement faciles à capturer. Ainsi bien souvent, le nom précède immédiatement le verbe, conduisant à une relation de contingence entre le « $\mathrm{s} »$ final du nom et le «nt » final du verbe. La proximité spatiale et/ou temporelle de ces marques plurielles est propice à l'apprentissage implicite de leur relation, et donc à la formation d'une nouvelle unité cognitive. Le résultat final est que l'accord peut se trouver correctement marqué, alors même qu'aucune analyse syntaxique n'est opérée, parce que la règle d'accord modifie des caractéristiques du matériel facilement appréhensibles par des mécanismes associatifs élémentaires.

Dans cet exemple, l'exploitation du trait perceptivement saillant corrélé à la règle d'accord conduit parfois à des erreurs. Elle conduit à ajouter «nt » dans la phrase « il les mange ». Ce genre d'erreur se trouve d'ailleurs fréquemment dans les productions des enfants, ou des adultes en situation de double tâche (Fayol, 2003; Fayol, Largy \& Lemaire, 1994). Ces erreurs confirment le fait que l'accord est souvent marqué grâce à des processus associatifs élémentaires reposant sur les relations entre traits de surface. Mais elles suggèrent également que cette approche est loin de conduire à des performances optimales. Cette conclusion a une part de vérité, sur laquelle nous reviendrons plus loin. De façon générale, cependant, cette limitation peut être levée s'il existe non pas un seul trait corrélé, comme dans l'exemple précédent, mais une multitude de traits corrélés, dont la considération conjointe viendrait palier les limites de chacun.

Ainsi, pour être viable, cette perspective requiert que l'analyse objective de domaines spécifiques révèle l'existence d'une pluralité de traits perceptivement saillants corrélés aux produits des règles formelles que le scientifique considère. Or il se trouve que, précisément, au moins en ce qui concerne le langage, de nombreuses recherches récentes sont orientées vers cet objectif, et rencontrent un succès grandissant. L'exemple le mieux documenté est certainement celui de la formation du passé en anglais. Il a été montré depuis longtemps que les verbes réguliers et irréguliers diffèrent en fonction de la distribution de leurs traits phonologiques. Par exemple, 
la majorité des verbes phonologiquement similaires à drink/drank ont également une flexion irrégulière (sing/sang, ring/rang, stink/stank). Mais la considération exclusive des traits phonologiques ne permet pas de résoudre tous les cas. Ainsi par exemple, comment expliquer qu'il soit possible de former le passé correct de verbes homophones (e.g., Brake and break) dont l'un est régulier (brake -> braked) et l'autre irrégulier (break -> broke) ? Ramscar (2002) a montré que ceci était possible, à la condition de considérer des indices additionnels, d'ordre sémantique. Des simulations connexionnistes ont montré que l'exploitation de ces indices corrélés conduit à une excellente approximation de la performance qui résulterait de la connaissance formelle de la règle du suffixe « ed », jointe à la connaissance des exceptions (e.g., McClelland $\&$ Patterson, 2002). Pour ne considérer qu'un autre exemple de la même approche, il a été montré que la considération de simples statistiques de co-occurrences (e.g., Redington, Chater, \& Finch, 1998) ou encore d'indices phonologiques (e.g., Monaghan, Chater, \& Christiansen, 2005), est riche d'informations sur les catégories grammaticales. Ces recherches suggèrent que les catégories abstraites sont souvent associées à des propriétés statistiques élémentaires qui les rendent appréhensibles par des mécanismes d'apprentissage associatif, et donc, comme montré plus haut, par les mécanismes d'auto-organisation de la conscience (Perruchet, 2005). Si les analyses à venir sur des corpus de langage confirment et étendent ces conclusions, et si le même type d'analyse se révèle heuristique dans d'autres domaines de compétences, alors le pari conceptuel que nous proposons se verrait très fortement conforté.

\section{Quelle pertinence pour l'éducation et la didactique?}

Il paraît nécessaire, avant d'aborder cette dernière section, de faire quelques mises en garde préliminaires. Il est toujours aventureux de vouloir tirer d'un modèle général du fonctionnement cognitif des implications éducatives, pour au moins deux raisons. La première est qu'un modèle, quel qu'il soit, reste une construction provisoire et spéculative, qui est appelée à évoluer, quand ce n'est pas à disparaître. La seconde raison est que, même si l'on est tenté d'accorder (à tort) une confiance indéfectible à un modèle donné, la distance entre des propositions générales sur le fonctionnement cognitif et leurs implications éducatives reste considérable. C'est donc en toute modestie que sont écrites les lignes suivantes, dont l'unique objectif est d'offrir quelques pistes de réflexion.

\section{Exploiter au mieux les propriétés du système....}

La position ici développée suggère qu'une composante essentielle de l'adaptation, à savoir la formation de représentations conscientes isomorphes au monde réel, peut s'opérer de façon spontanée, grâce à des processus d'auto-organisation, par le jeu des interactions naturelles entre le sujet et son environnement. En un sens, donc, ce système de pensée conduit à relativiser le poids des processus de compréhension, ou du moins à considérer que des activités orientées vers une meilleure compréhension du monde et de ses lois ne peuvent être conçues comme les seules activités réellement éducatives. Il ne s'agit pas ici de renforcer le poids de l' " apprentissage par cœur » (qui peut garder par ailleurs toute sa valeur), mais de réaliser que la dichotomie classique opposant compréhension et mémorisation laisse sans doute de côté une composante essentielle, à savoir la formation d'unités de pensée dont la pertinence conditionne dans une large mesure l'efficacité des processus de compréhension, aussi bien d'ailleurs que celle des processus de mémorisation. Certes, ces unités devraient se développer d'elles-mêmes, pour peu que l'élève soit placé dans un environnement structuré avec lequel il interagit activement, quelle que soit la finalité déclarée de l'activité. Il ne s'agit pas pour autant de nier l'importance du choix de cette activité. L'un des rôles de l'éducateur pourrait être de favoriser l'élaboration d'unités cognitives adaptées (1) en guidant l'attention de l'élève vers les éléments composant ces unités, (2) tout en évitant les conditions propices à la formation d'associations parasites. Chacun de ces deux aspects sera considéré en succession.

Pour illustrer comment l'attention de l'élève peut être guidée vers les éléments à associer, sans pour autant expliciter ces derniers, considérons un exemple issu de l'apprentissage de l'écriture, en particulier de lettres cursives. Lorsqu'un expert produit une lettre cursive, il a été montré qu'une relation remarquable existe entre le niveau cinématique de description du mouvement et le niveau géométrique de descrip- 
tion du tracé de la lettre : chaque segment de lettre compris entre deux points d'inflexion de la trajectoire est produit par un couple accélération-décélération, donc par une unité motrice (voir Zesiger, 1995). Ainsi la lettre cursive « $l$ », par exemple, peut être décomposée en trois segments remarquables, un premier segment courbe ascendant, un second segment rectiligne descendant et un troisième petit segment ascendant. L'enfant, au début de son apprentissage, va produire cette même lettre en recrutant un nombre considérable d'unités motrices, chaque segment remarquable étant alors formé de plusieurs sous-segments, avec des arrêts et des changements de direction locaux (Zesiger, 1995). Développer une écriture fluide reviendra à apprendre à produire une trajectoire plus longue en conséquence d'une accélération et d'une décélération du mouvement, tout en respectant la forme de la trajectoire visée. Face à la lettre cabossée produite par l'enfant novice, on peut évidemment penser l'aider à améliorer son mouvement en lui montrant toutes les hésitations et les changements de direction inappropriés, en lui montrant le moment où le changement de direction est nécessaire, en d'autres termes, en analysant explicitement la distance entre sa production et le modèle. Cette approche a toutefois pour inconvénient d'inciter l'enfant à faire de fréquents reports entre sa production et le modèle, ce qui est directement préjudiciable à la fluidité de son mouvement, ces reports impliquant nécessairement des arrêts et ruptures dans le mouvement. Dans une recherche récente, nous avons testé l'effet de l'association de deux contraintes, l'une temporelle (accélérer le mouvement), l'autre spatiale (produire la lettre à l'intérieur d'interlignes marquant les moments de changements de direction), sur la production de lettres cursives par le jeune enfant, avant l'apprentissage systématique de l'écriture cursive (Chartrel \& Vinter, sous presse). La première contrainte a pour effet d'amener incidemment l'enfant à produire une plus longue trajectoire au moyen du recrutement d'un seul couple accélération-décélération, la seconde a pour effet de contenir l'augmentation de vitesse (afin de minimiser les déformations de la lettre) et surtout de faciliter la planification $\mathrm{du}$ mouvement en marquant incidemment, par les interlignes, les points de changements de direction. Les résultats obtenus sont encourageants, la fluidité du mouvement de l'enfant pré-scripteur augmente dans ces conditions de contraintes spatio-temporelles, mais il faut mentionner toutefois l'incapacité du jeune enfant à produire, sous ces contraintes, les lettres cursives aux formes difficiles, comme le $\mathrm{k}$ ou le g par exemple.

Abordons maintenant le deuxième point soulevé précédemment, la formation éventuelle d'associations parasites. Éviter les conditions propices à la formation d'associations parasites peut sembler un conseil inutile, tant cela parait relever du bon sens. C'est oublier que certaines méthodes d'enseignement ou de test peuvent indirectement créer ces conditions. Il en est ainsi des activités conduisant à des erreurs, telles que les activités orientées vers la découverte d'une réponse correcte parmi un ensemble de réponses proposées, ou de façon plus générale les activités privilégiant la recherche active d'une solution à l'énoncé direct de cette solution. Un problème potentiel de ces méthodes est que les apprenants peuvent se familiariser aussi bien avec les associations erronées qu'avec les associations correctes (Baddeley \& Wilson, 1994; Fischer, 1999). Dans le domaine de l'orthographe, par exemple, il a pu être montré que des pratiques conduisant à favoriser la production d'erreurs à des fins de comparaison avec la forme orthographique correcte, ou exposant l'élève à des mots mal orthographiés au sein d'une épreuve à choix multiples servant de test, avaient un effet délétère (e.g. Brown, 1988; Dixon \& Kaminska, 1997; Rey, Pacton, \& Perruchet, 2005). Ces effets sont loin d'être négligeables, et l'on a pu montrer qu'ils s'exerçaient même lorsque l'apprenant sait parfaitement que l'information qui lui est présentée est fausse (Perruchet, Rey, Hivert, \& Pacton, 2006). En d'autres termes, ils s'exercent même, par exemple, quand l'élève connaît la solution correcte dans une épreuve à choix multiples.

Faut-il en conclure que ce type d'épreuve, et de façon générale toutes les méthodes d'apprentissage ou de test exposant l'élève à l'erreur, doivent être bannies? Pas nécessairement. L'efficacité d'une méthode donnée ne peut être évaluée que globalement. Ainsi par exemple, une façon d'éviter les erreurs consiste à répéter immédiatement un énoncé ou un exercice, plutôt que de laisser à l'oubli le temps de s'instaurer. Toutefois, cette pratique va à l'encontre d'un autre principe, qui est que les répétitions espacées par un intervalle temporel sont plus efficaces pour la mémorisation à long-terme que les répétitions «massées» (pour une revue : Perruchet, 1987). 
Pashler, Zarow, et Triplett (2003) ont montré qu'en cas de conflit, il valait mieux espacer les répétitions, au risque d'accroître la probabilité d'erreur. Un autre exemple est l'usage de tests à choix multiples à des fins d'évaluation. Ces tests exposent inévitablement l'élève à des erreurs, mais ils ont également, du moins s'ils sont adéquatement construits, des conséquences positives pour la consolidation des connaissances. Au terme d'une revue de question sur le sujet, Marsh, Roediger, Bjork, et Bjork (2007) concluent que l'effet bénéfique des tests à choix multiples semble généralement prévaloir sur les conséquences négatives. Enfin, il est évident pour chacun que l'exposition à des erreurs, ou leur production, peut avoir un rôle pédagogique pour aider à la compréhension. Cet aspect a été suffisamment souligné pour qu'il soit inutile de le développer davantage. C'est donc « toutes choses égales par ailleurs » que le risque inhérent à l'exposition à l'erreur doit être pris en considération.

Une caractéristique généralement attribuée aux formes implicites d'apprentissage est qu'elles apparaissent relativement préservées lorsque les capacités cognitives générales, et notamment les possibilités d'apprentissages explicites, se trouvent dégradées par l'âge ou par des troubles neurologiques (par exemple, Vinter \& Detable, 2003). Ceci suggère que certains prolongements des idées exposées plus haut pourraient se révéler utiles dans les situations de rééducation. Il faut reconnaître que la littérature est particulièrement silencieuse à ce sujet, et à notre connaissance, il n'existe toujours pas de travaux référencés ayant démontré l'efficacité d'une rééducation par apprentissage implicite. Nous ne pouvons que suggérer des pistes de réflexion à partir de nos travaux fondamentaux. Nous avons, par exemple, montré qu'il était possible de faire acquérir, de manière implicite, un nouveau comportement graphique, aussi bien chez des adultes (Vinter \& Perruchet, 1999) que chez des enfants (Vinter \& Perruchet, 2000, 2002). Ce nouveau comportement graphique consistait à associer le point de départ du tracé d'une forme géométrique (comme un cercle par exemple) et la direction de la rotation du mouvement (horaire ou antihoraire) de manière inverse à ce que, spontanément, chacun d'entre nous produit. Si une telle association peut être établie par une procédure appropriée d'apprentissage implicite, alors il peut être suggéré qu'une procédure similaire pourrait être utilisée dans le cadre de rééducation de l'écriture, pour apprendre aux enfants en difficulté à produire les lettres en respectant un point de départ et un sens de rotation donnés.

\section{... En restant conscient de ses limitations}

Au chapitre des limitations, il en est une première qui relève de la pertinence des unités perceptives et représentationnelles qui découlent naturellement du traitement attentionnel de l'environnement. Nous avons insisté sur le fait que ces unités sont plus pertinentes et plus complexes que ce que l'on pourrait attendre de simples mécanismes associatifs prenant pour base des éléments perceptivement saillants. Il convient toutefois d'ajouter qu'il existe des exceptions notoires. Ainsi la représentation de l'espace que l'homme peut se former par l'observation du ciel nocturne est évidemment inexacte. Les unités perçues et décrites sous la forme de constellations se composent d'étoiles qui peuvent être situées à des milliers d'années-lumière les unes des autres du fait que les distances entre objets célestes sont déformées par leur projection sur la voûte céleste. Ces erreurs, considérables lorsqu'elles sont évaluées en regard de la connaissance scientifique, sont évidemment sans conséquence au niveau de l'adaptation comportementale - tant qu'il ne s'agit pas d'envoyer une fusée dans l'espace -. Cet exemple illustre toutefois que la connaissance du monde qui pourrait être inférée des représentations façonnées par des mécanismes d'apprentissage implicite peut se révéler totalement erronée.

Ces limitations ont plusieurs causes. Dans l'exemple précédent, elles proviennent essentiellement de l'incomplétude des informations perceptives disponibles. Mais même lorsque les informations pertinentes sont disponibles, il reste que l'apprentissage implicite ne peut détecter que certaines structures. Par exemple, il est difficile de détecter des relations entre éléments distants, spatialement ou temporellement. Certaines recherches de laboratoire ont démontré que les éléments à associer n'ont pas besoin d'être strictement contigus (e.g. Gomez, 2002), mais que la possibilité d'établir une association semble décroître très rapidement quand la distance entre les éléments s'accroît (Cleeremans, 1993). Le rôle essentiel de l'attention dans la formation d'un apprentissage laisse penser que les éléments à associer doivent être 
perçus dans un même focus attentionnel, dont on sait l'empan étroitement limité. Très vraisemblablement, cet empan est insuffisant pour apprendre un certain nombre de relations utiles. Considérons à nouveau le cas de l'orthographe. Les formes implicites d'apprentissage sont particulièrement efficaces pour des régularités graphotactiques, concernant par exemple l'influence du contexte consonantique droit et/ou gauche sur l'écriture de la voyelle adjacente (Pacton, Fayol \& Perruchet, 2002). Sans doute est-il possible d'apprendre sous ce mode, avant même d'avoir reçu une instruction explicite (Pacton \& Fayol, 2003), des associations plus distantes, telle que la terminaison « ant " après « en ...» pour marquer le gérondif. Mais certaines règles de l'orthographe portent sur des dépendances beaucoup plus éloignées, qu'il est sans doute plus difficile d'apprendre sans passer par l'acquisition de connaissances explicites. Dans ce dernier cas, l'apprentissage implicite peut conduire à des erreurs. Il arrive en effet que l'application d'une règle exige que l'on aille à l'encontre des régularités statistiques et des unités cognitives qui en découlent. L'exemple cité plus haut de l'association entre la forme plurielle d'un verbe et la forme plurielle du mot qui le précède immédiatement illustre parfaitement le point. Pour écrire correctement « il les mange » et non « il les mangent » ou « il les manges », il faut aller contre une régularité statistique en recourant à la règle d'accord que la grammaire stipule.

Ce risque d'erreur, toutefois, ne doit pas être exagéré. Tant qu'il s'agit d'ajustements comportementaux, les situations dans lesquelles les effets de l'apprentissage implicite doivent être contrecarrés sont sans doute l'exception, ou en tous cas ne constituent vraisemblablement que des situations dans lesquelles les erreurs sont dépourvues de conséquences vitales. En effet, si les régularités statistiques n'étaient pas un bon guide pour ajuster le comportement au monde environnant, des mécanismes orientés vers leur exploitation n'auraient pas été sélectionnés au cours de l'évolution. La théorie synthétique de l'évolution conduit à prédire que dans la plupart des cas, l'apprentissage implicite génère des représentations du monde conduisant elles-mêmes à des réponses conformes à celles auxquelles conduit le respect des règles.

Il existe toutefois une autre limitation. Les unités perceptives et représentationnelles formées par auto- organisation nous procurent une façon de voir le monde et d'interagir avec lui. Elles ne peuvent être assimilées à une " connaissance sur » le monde. Disposer d'unités cognitives pertinentes est sans doute nécessaire, mais ne peut être conçu comme suffisant. Limportance éducative de ce point ne peut être sous-estimée. Apprentissage implicite et enseignement scolaire ne peuvent être conçus comme des méthodes alternatives visant l'atteinte d'un même objectif. Cette idée reste fausse même si l'on considère que les connaissances résultantes sont implicites dans un cas et explicites dans l'autre cas. Ce constat va à l'encontre d'une perspective fréquente selon laquelle, dans certains domaines au moins, l'apprentissage scolaire permettrait l'explicitation de connaissances que l'enfant aurait déjà acquises implicitement. Il s'agirait essentiellement de prise de conscience : rendre explicite une connaissance tenue tacite jusqu'alors. Ce scénario, basé sur l'existence d'une continuité entre les premières adaptations comportementales et les connaissances formelles ultérieures, peut trouver support dans certaines théories développementales (e.g. Karmiloff-Smith, 1992). Toutefois, les travaux de laboratoire sur l'apprentissage implicite vont à l'encontre de ces théories (Perruchet \& Vinter, 1998). Si l'on donne crédit à ces travaux, il nous faut admettre que les apprentissages implicites ne conduisent pas à la connaissance implicite des règles que l'école pourvoit de façon explicite: ils reposent sur des formes adaptatives alternatives. Les règles en tant que telles ne sont pas internalisées, c'est l'apprenant qui se forge des représentations internes du monde dépendantes, de par leur mode de formation, de caractéristiques distributionnelles de traits perceptivement saillants, caractéristiques qui sont elles-mêmes une conséquence plus ou moins directe des règles. Linstruction scolaire apporte quelque chose de différent par rapport à ce à quoi conduisent les processus d'apprentissages implicites et d'auto-organisation. Parce que l'apprentissage implicite ne peut conduire, de par sa nature, à l'établissement de connaissances formelles, structurées par des règles abstraites, et aisément communicables, il ne peut remplir l'ensemble des objectifs habituellement conférés à l'enseignement. C'est pourquoi nos connaissances actuelles invitent à penser l'articulation entre apprentissage implicite et enseignement scolaire davantage en termes de complémentarité qu'en termes de substitution. 


\section{BIBLIOGRAPHIE}

Baddeley, A.D. \& Wilson, B.A. (1994). When implicit learning fails: Amnesia and the problem of error elimination. Neuropsychologia, 32, 53-68.

Brent, M.R. (1996). Advances in the computational study of language acquisition. Cognition, 61, 1-38.

Brown, A.S. (1988). Encountering misspellings and spelling performance: Why wrong isn't right. Journal of Educational Psychology, 80, 488-494.

Chartrel, E. \& Vinter, A. (sous presse). The impact of spatio-temporal constraints on cursive letter handwriting in children. Learning and Instruction.

Clark, A. \& Thornton, C. (1997). Trading spaces: Computation, representation and the limits of uninformed learning. Behavioral and Brain Sciences, 20, 57-90

Cleeremans, A. (1993). Mechanims of Implicit Learning: A connectionnist model of sequence processing. MIT press: Bradford Books (pp.227)

Deneubourg, J-L., Aron, S., Goss, S. \& Pasteels, J.M. (1990). The self-organizing exploratory pattern of the Argentine ant. Journal of Insect Behavior, 3, 159.

Dixon, M. \& Kaminska, Z. (1997). Is it misspelled or is it mispelled? The influence of fresh orthographic information on spelling. Reading and Writing, 9, 483-498.

Evans, J.St.B., Newstead, S.E., \& Byrne, P. (1993). Human reasoning. Hillsdale, N.J.: Erlbaum.

Fayol, M. (2003). Lacquisition/apprentissage de la morphologie du nombre. Bilan et perspectives. Rééducation Orthophonique, 213, 151-166.

Fayol, M., Largy, P. \& Lemaire, P. (1994). Subject- verb agreement errors in French. Quarterly Journal of Experimental Psychology, 47A 437-464.

Fischer, J.P. (1999). Le rôle exclusivement positif des erreurs: une conception erronée de l'apprentissage. Résonances, 5, 9-11.

Gomez, R. (2002). Variability and detection of invariant structure. Psychological Science, 13, 431-436.

Karmiloff-Smith, A. (1992). Beyond modularity: A developmental perspective on cognitive science. Cambridge, MA: Bradford/ MIT press.

Marsh, E. J., Roediger, H. L., Bjork, R. A. \& Bjork, E. L. (2007). The memorial consequences of multiple-choice testing. Psychonomic Bulletin \& Review, 14, 194-199.

McClelland, J. L. \& Patterson, K. (2002). Rules or connections in past-tense inflections: What does the evidence rule out? Trends in Cognitive Sciences, 6, 465-472.

Monaghan, P., Chater, N. \& Christiansen, M.H. (2005). The differential role of phonological and distributional cues in grammatical categorisation. Cognition, 96, 143-182.

Pacton, S. \& Fayol, M. (2003). How do French children use morphosyntactic information when they spell adverbs and present participles? Scientific Studies of Reading, 7, 273-287.

Pacton, S., Fayol, M. \& Perruchet, P. (2002). The acquisition of untaught orthographic regularities in French. In : L. Verhoeven, C. Erlbro \& P. Reitsma (Eds.), Precursors of Functional Literacy. Dordrecht: Kluwer.

Pacton, S. \& Perruchet, P. (sous presse). An attention-based associative account of adjacent and nonadjacent dependency learning. Journal of Experimental Psychology: Learning, Memory, and Cognition.

Pashler, H., Zarow, G., \& Triplett, B. (2003). Is temporal spacing of tests helpful even when it inflates error rates? Journal of Experimental Psychology: Learning, Memory, \& Cognition, 29, 1051-1057.

Perruchet, P. (1987). Pourquoi apprend-on mieux quand les répétitions sont espacées ? Une évaluation des réponses contemporaines, Année Psychologique, 87, 253-272.

Perruchet, P. (2005). Statistical approaches to language acquisition and the self-organizing consciousness: A reversal of perspective. Psychological Research, 69, 316-329. 
Perruchet, P. \& Pacton, S. (2004). Qu'apportent à la pédagogie les travaux de laboratoire sur l'apprentissage implicite? Année Psychologique, 104, 121-146.

Perruchet, P. \& Pacton, S. (2006). Implicit learning and statistical learning: Two approaches, one phenomenon. Trends in Cognitive Sciences, 10, 233-238

Perruchet, P., Rey, A., Hivert, E. \& Pacton, S. (2006). Do distractors interfere with memory for study pairs in associative recognition? Memory and Cognition, 34, 1046-1054.

Perruchet, P. \& Vinter, A. (1998a). PARSER: A model for word segmentation. Journal of Memory and Language, 39, 246-263.

Perruchet, P. \& Vinter, A. (1998b) Learning and development: The implicit knowledge assumption reconsidered. In M. Stadler \& P. Frensch (Eds), Handbook of Implicit Learning (pp. 495-531). Thousand Oaks, CA: Sage Publications.

Perruchet, P. \& Vinter, A. (2002). The Self-Organizing Consciousness (Target paper). Behavioral and Brain Sciences, 25, 297- 330.

Perruchet, P. \& Vinter, A. (sous presse). La conscience auto-organisatrice. Année Psychologique.

Ramscar, M. (2002). The role of meaning in inflection: Why the past tense does not require a rule. Cognitive Psychology, 45, 45-94.

Redington, M., Chater, N. \& Finch, S. (1998). Distributional information: A powerful cue for acquiring syntactic categories. Cognitive Science, 22, 425-469.

Rey, A., Pacton, S. \& Perruchet, P. (2005). L'erreur dans l'acquisition de l'orthographe. Rééducation Orthophonique, 222, 101-120.

Vinter, A. \& Detable, C. (2003). Implicit learning in children and adolescents with mental retardation. American Journal on Mental Retardation, 108, 94-107.

Vinter, A. \& Perruchet, P. (1999) Isolating unconscious influences: The neutral parameter procedure. Quarterly Journal of experimental Psychology, 52A, 857-875.

Vinter, A \& Perruchet, P. (2000). Implicit learning in children is not related to age : Evidence from drawing behavior. Child Development, 71, 1223-1240.

Vinter, A. \& Perruchet, P. (2002). Implicit motor learning through observational training in adults and children. Memory and Cognition, 30, 256-261.

Zesiger, P. (1995). Ecrire: approches cognitive, neuropsychologique et développementale. Paris: PUF. 